\title{
Mobile Market Research Applications as a New Voice of Customer Method
}

\author{
A Qualitative Study of the Implications for Innovation and Design \\ Management
}

William Green, Robert Cluley, and Marta Gasparin

\section{Bios below}

William Green is an associate professor at the University of Leicester School of Business. He researches design and innovation management, human factors, and the role of new technology in the interface between people, technology, and their environment. This research has led to recent studies of innovation and technology in the healthcare and consumer research sectors, investigating the impact of new technology on practice. w.green@leicester.ac.uk

Robert Cluley is an associate professor at the University of Nottingham Business School. His research, exploring the use of technology and data in marketing practice, has been published in leading journals, including Marketing Theory, Organization Studies, and Industrial Marketing Management. He is the author of Essentials of Advertising (Kogan Page, 2017).robert.cluley@nottingham.ac.uk

Marta Gasparin is an associate professor at the University of Leicester School of Business. Her research explores the emergence of design and innovation, value creation, and the role of human and nonhuman actors in innovation processes. She is leading an ESRC-funded project, Slow Design-Driven Innovation, in the UK and Vietnam. marta.gasparin@leicester.ac.uk

Overview: This paper describes mobile market research applications (MMRAs), new Voice of Customer (VoC) tools that take advantage of the ubiquity of smart phones. MMRAs provide an efficient and effective method of collecting intimate, in situ data from customers, allowing traditional $\mathrm{VoC}$ approaches to be augmented with digitally captured data. MMRAs can thus reduce ambiguity in the fuzzy front end of the innovation process by providing access to data from likely customers from the earliest stages of exploration. To benefit from MMRAs, however, organizations must move beyond a traditional mindset: the adoption of an MMRA requires teams to be restructured and projects reorganized to accommodate the new methods enabled by the tools.

Keywords: Voice of the Customer; Design research; Digital tools 
Voice of the Customer (VoC) methods provide new product development teams with tools to capture customers' preferences and needs so that they can develop products that satisfy those preferences and needs. Traditionally, VoC methods have provided customer input into product conceptualization and development through techniques such as indepth, in-person interviews; focus groups; and ethnographic studies. These approaches allow new product development teams to get close to customers at the point at which they interact with products. However, these approaches can be time-consuming and costly, and the data they produce can be difficult to translate into practice.

Big data and marketing analytics have offered tools to overcome these issues, by making it possible to amass large data sets quickly and at low cost. For example, social media listening allows companies to observe what consumers are saying about them online, capturing a general sense of consumers' sentiment, tone, and emotions. Yet, questions persist regarding the ability of these methods to authentically represent the customer voice as, unlike traditional methods, they remove the researcher from the action and do not allow customers to communicate directly. They also may suffer from a lack of focus, as these techniques tend to be used to collect data from consumers passively and without having been designed to accomplish specific research purposes.

A new VoC method, mobile market research applications (MMRAs), takes advantage of the ubiquity of smartphones to actively collect large volumes of in-depth data through an app designed with the specific purposes of the research in mind. MMRAs also make the communication two way, allowing consumers to express their opinions and communicate their experiences through videos, notes, games, and tasks - all without face-to-face interaction with a researcher. Customers' interaction with the app produces large-scale data sets that represent an authentic customer response to the research question. As a result, MMRAs can deliver the benefits of big data approaches while overcoming their limitations, combining the intimacy of traditional $\mathrm{VoC}$ with the efficiency of big data approaches.

\section{The Voice of the Customer in New Product Development}

The objective of $\mathrm{VoC}$ is to bring the customer's voice into the new product development process. VoC methods allow customers to express themselves freely. VoC data can reveal unmet, hidden needs (Cooper and Edgett 2008; Goffin, Lemke, and Koners 2010), and studies have shown that they drive innovation (Cooper and Edgett 2008) and contribute to the development of radically innovative products (Davila, Epstein, and Shelton 2012).

VoC methods traditionally include qualitative and quantitative approaches. Qualitative research methods, such as ethnographic studies, customer journey research, and in-depth interviewing, provide rich data about particular customers and their individual experiences and rituals. Quantitative research methods, such as large-scale surveys, provide opportunities to model the past and future behaviors of whole segments of a market. The choice between methods is ideally driven by the questions that need to be answered. But in practice, the choice is often driven by trade-offs and limitations in available time, money, and expertise. 
Big data and marketing analytics promises to revolutionize the types of data used in new product development, because they overcome some of the traditional VoC limitations and trade-offs. By automating the process of data collection, which has traditionally been carried out in person, big data-based methods reduce the costs and time involved in VoC research. Data such as transactional records, social media posts, and location data can be continually harvested and analysed for patterns that provide clues to consumers' preferences and needs. These data can be collected passively; customers may not even be aware of the collection, let alone conscious of its purpose (Cluley and Green 2019). These data can be collected efficiently in huge amounts and then segmented into clusters, allowing customer behavior to be modeled using predictive analytics. Yet, despite wide adoption of these methods, they have not yet been shown to contribute to the development of successful new products. Indeed, the power and challenge of using data gathered through wearable technology, mobile communication devices, virtual reality, social media activities, and augmented reality have been well publicized and widely explored (George, Haas, and Pentland 2014).

Passively collected data can be messy, uncertain, and ambiguous because it tends to be generated with "no specific question in mind" (Kitchin 2014, p. 2). As a result, managing, analyzing, and incorporating into an innovation the information the data provide. Furthermore, the volume of data may come at the cost of losing in-depth, individual-level customer insights. Big data can reveal hidden patterns in customer datasets, but it does not allow customers to express their motivations, desires, fears, hopes, dreams, or experiences. Even rating or recommender systems, which allow for some individual customer expression, must do so within a limited structure. Gobble (2015) argues that, even in the age of big data, companies need to develop intimate knowledge about customers. Big data, by its very nature, can make it impossible to achieve that intimacy.

However, these limitations are not inherent to digital VoC methods; these technologies can be used to actively collect data to answer specific research questions in ways that allow customers to voice their needs and preferences. Actively collected data provides an exciting opportunity to overcome the trade-offs in VoC practices. Active data collection can be targeted to gather data on large numbers of people in a specified population, and it can allow customers to describe their needs in their own words, providing the intimate knowledge companies need to identify and satisfy hidden customer needs. It can reduce ambiguity in the fuzzy front end of the innovation process by focusing the project team on real customer needs.

MMRAs provide a conduit for such data collection. MMRAs use smartphones to facilitate active data collection and direct engagement with consumers via apps that ask customers to express their experiences and opinions in videos, notes, games, tasks, sound recordings, and photographs. While mobile apps have long been used to collect data passively, as consumers engage in other tasks, the use of mobile apps designed specifically for research purposes emerged in the market research industry in the early 2010s. One early MMRA platform was created by a collaboration between the University of Leicester School of Business and a design firm (Green and Cluley 2014). It asked users of the app (customers) to complete tasks designed by researchers to answer specific 
questions, such as reporting their mood at key points in the customer experience (Figure 1). An MMRA allows researchers to define the questions they need to ask and access authentic customer voices without their physical presence. Thus, MMRAs can enable VoC efforts to produce large volumes of data that reflect authentic customer needs and experiences in a cost-effective and timely manner. MMRAs may be custom designed for a given project, built on a proprietary system owned by the company, or adapted (or adopted) from existing tools or frameworks.

\section{---Figure 1 near here---}

Given the paucity of information regarding the effect of MMRAs on product development, we set out to explore whether MMRAs can provide a suitable alternative, or complement, to traditional VoC methods.

\section{Method}

Over a two-year period, the primary author captured practitioner perspectives to test the thesis that MMRAs provide a VoC research method that combines the benefits of big data with the strengths of traditional VoCs. (The study was part of a larger exploration of MMRAs undertaken in collaboration with a digital design firm.) Participants were recruited to participate in the study on the basis of their experience of MMRAs. The study sample included client-side organizations $(n=19)$, which commission $R \& D$ and marketing agencies to conduct the research, and research providers $(n=25)$; all participants were early adopters of MMRAs. Access to these organizations was brokered by an MMRA development company that was interested in MMRA lead users' experiences as a part of their innovation process. They had developed a bespoke MMRA as their main product, their clients range from small regional companies to multinational brands.

Researchers interviewed participants using an interview protocol to guide queries about interviewees' experience with MMRAs. The protocol focused on exemplary MMRA projects and the challenges and benefits interviewees perceived in using MMRAs. All interviews were anonymized. Twenty-nine of the interviews were transcribed and managed within QSR International's NVivo software. The remaining fifteen were not recorded due to commercial sensitivities. For those interviews, data collection was confined to note taking. Two of the authors thematically coded the dataset to test the thesis that MMRAs can be used for VoC research. The findings of the research fed into an MMRA product development cycle at the partner organization.

\section{Findings}

All respondents reported successful implementation of MMRAs in a variety of development projects, including both service and product design projects for small and medium-sized enterprises and large corporations. For example, one project used an MMRA to follow customers from home, through the airport, to their holiday or business destination (see "Case 1: Exploring Travelers' Experiences to Drive an Airlines' Strategic Design Decisions," below). A multinational coffee brand used an MMRA to invite customers to capture their coffee-drinking experiences through a range of media, as 
part of an effort to redesign the coffee cup (see "Case 2: Reconceptualizing the Experience of Coffee Drinking," below). And a multinational dental hygiene company used an MMRA to understand childrens' toothbrushing practices (see "Case 3: Mapping Children's Experiences of Toothbrushing," below).

\section{---Text box 1 ("Case $1 \ldots$. . ) near here--- \\ ---Text box 2 ("Case $2 \ldots$. . ) near here--- \\ ---Text box 3 ("Case $3 \ldots$. . ") near here---}

Interviewees reported finding MMRAs to be generally effective and more resourceefficient than traditional VoC methods. As a digital research manager at a client-side organization told interviewers, "You can really get very nice, very visual stuff built up, without us actually going there. And without us even interviewing them in person. Just from getting a really well-designed question structure." All interviewees reported using MMRAs to aggregate customer responses in real time, expose customer needs, and in some cases solicit customers' evaluations of both prototypes and existing products. Some researchers even provided clients access to the apps' dashboard, so clients could see data as they were collected.

The interview data provide evidence of many benefits of MMRAs over traditional VoC methods. The benefits primarily concern the quality, accessibility, and shareability of the resulting data. MMRAs allow data to be collected via a variety of media - survey responses, text comments, videos, voice recordings, geospatial data, and images, among others. They also enable customers to provide real-time data at any point in their experiences. In the airline case, for instance, the MMRA approach allowed researchers to collect data that would have been extremely difficult to access any other way. Having researchers physically follow participating passengers on their journeys would have been logistically challenging, to say the least, especially as passengers moved from private to public and regulated spaces in an airport. A passenger's smartphone, however, can travel with him or her throughout the journey. Thus, smartphones could collect data when researchers might have had to rely on retrospective reports or other methods.

The benefits also relate to the ways in which MMRAs empower customers to voice their experiences when and how they see fit; because a researcher is not physically present, the data may be more authentic. Participants decide what to share and what form to give to their experiences. One of our interviewees, a digital research manager, explained that the organization used MMRAs purposively to design different enjoyable tasks: "We want to bring something different, basically, we want to make it fun, and we want to make it like a game for them . . . because usually when we do a quant [traditional survey research], it's like we get a bunch of answers, but when you think about the why, it's hard to understand." The interviewee found that research participants are more likely to provide authentic information about their experiences and opinions when they find a task rewarding or enjoyable. 
In addition, because digital data are easy to share, MMRAs improve researchers' ability to communicate VoC data within the development team and across the organization. Digital data can be shared in the cloud, sent via email, or transmitted wirelessly or via physical data-storage devices. Further, data can be shared in raw form or after analysis, so teams can access unmediated customer voices. This ease of shareability can also streamline workflows. In the toothbrushing case, a traditional approach would have required the design team to synthesize reports from separate research teams, but the MMRA allowed all of the data to be analyzed together by a central research team.

A final benefit of the MMRA approach that all interviewees discussed concerned research management. The method is cost-effective and efficient, providing the ability to gather a large dataset more quickly than can be done with traditional methods. Further, customers' engagement with tasks can be tracked in the MMRA, allowing researchers to prompt responses and engage in other tactics to improve response rates and increase data quality. And, as one interviewee who works in a design agency, pointed out, the tracking ability can let researchers see whether their ideas for data collection tasks are working: "So we wanted to check that the idea was actually going to work, were people, could they get their husband or child to film them, and we'd get actual quality footage rather than someone just going and missing it completely." A task that isn't yielding the expected data can be redesigned or replaced-while the study is in progress.

These abilities also feed into another benefit of MMRAs: the ability to assess engagement and data quality during data collection and send "top line" findings and interesting observations to managers, colleagues, and clients as the study proceeds. These regular updates and emerging findings, interviewees reported, could create excitement and anticipation and build support for the work. One interviewee, a research manager, described an instance in which the client, who traditionally would not have interacted with the research team between commissioning the research and receiving the final research report, asked for further information while data collection was ongoing. "I think definitely for a client, the [top line's] powerful. It means if they [the client] are being really lazy, one look and they've got some really nice insight there and some quotes ... the client rang up and said who is the star responder . . . can you tell us a bit more about him."

\section{Challenges in Implementing MMRAs}

Interviewees identified one overarching challenge in the MMRA approach, emerging from the tool's biggest advantage: the richness and quantity of data MMRAs can provide. In some projects, interviewees reported, the MMRA delivered an overwhelming amount of data, more than researchers could analyze in the time allocated to the project. Some researchers responded to the overwhelm by analyzing data continuously as it rolled in, working evenings and weekends to keep up. As one interviewee reported: "I had to work late evenings and work a number of weekends to get all the data reviewed...there just wasn't enough days in the week." Others reported using only a small sample of a large data set. In other words, while MMRAs provide a cost-effective way of gathering large data sets, the volume of data can create additional costs in analysis. If researchers and clients do not allow sufficient time, individual researchers may struggle to cope, leading 
to burnout, and projects may fail to unlock all potential insights when data are summarized, extracted, or ignored.

Similarly, early reporting of initial findings can generate excitement for the project, but it can also create challenges. Early analysis can lead to premature decisions or anchoring to initial findings that should be modified or even discarded in the context of the full analysis. At the same time, the push to provide meaningful findings even during data collection can pressure researchers to generate additional prompts in the quest for interesting and novel data. Too many prompts could undermine customers' sense that they can freely voice their experiences, interfering with the unmediated VoC MMRA can provide. They could also lead to customers becoming frustrated and disengaging from the research.

An MMRA approach may also challenge traditional divisions between qualitative and quantitative research teams. Analyzing the data collected by an MMRA requires drawing on both qualitative and quantitative methods, forcing teams to work across disciplines. The ability to collect data in a variety of forms, as in the coffee drinking case above, allowing participants to respond using different media, means that MMRA projects cannot always follow traditional analytical methods. Researchers may be required to engage in semiotic analysis of videos and photographs, content analysis of notes and text entries, and quantitative analysis of tasks, games, surveys, and locational data. Combining these different response forms to reveal the voice of the customer requires a flexible approach to analysis, which, in turn, requires a flexible approach to team management.

Respondents in established research organizations reported that achieving this flexibility can be difficult, because these organizations tend to be structured around specialist teams, with each team focused on a particular method. Organizations that are succeeding with MMRAs find they have to relax these boundaries to maximize insights. Indeed, other boundaries are being relaxed, as well. One interviewee at a research organization reported that, while he predominantly works in the operational side of projects, he is having to "get involved in the research side of the projects as well" in order to meet project deadlines. She continued, "So I started to analyze the stuff, looking at the data and doing top-line reports, so, although predominantly I'm from operations, I'm starting to do, step by step, the research side of it as well."

MMRAs can also present challenges for individual researchers. MMRA projects are stressful for researchers for a number of reasons. One, of course, is the resources needed to manage high-volume data flows and provide timely analysis. Another source of stress is the design of the studies themselves. MMRA-based studies often involve designing customized, innovative tasks that can fail to provide the data sought. Often, the MMRA tasks also demand new ways of analyzing data that do not fit established processes and protocols. Individual researchers also reported time pressures because they were expected to analyze data while monitoring collection. For example, one researcher commented on the need to check data as they were collected: 
If you wait until the end of the project and [a research participant has] not done what they're meant to do - even if they have been doing something, [the researchers] need to $\log$ on and check that [the research participant is] doing the right thing. They [the research participant] ... could have completely got the wrong end of the stick, or whatever. Or be reading the questions wrong ... the researchers that I've worked with do definitely check it and are almost analyzing data as it goes along.

A final challenge of MMRA is research ethics, notably with regard to the sharing of raw data. A large proportion of market research is conducted by specialist agencies. Those agencies typically enter into agreements with research participants that prohibit sharing of raw data with the agency's clients. The quality and richness of MMRA data have led clients to pressure researchers for access to raw data, to get closer to the authentic voice of the customer, to share among their internal teams, or to use for other purposes. Acceding to these demands often requires researchers to ask customers for further permissions, a request that can stress relationships with VoC participants.

\section{Discussion}

MMRA methods undoubtedly allow innovation practitioners intimate access to the customers voice. Research teams and clients in our study unanimously considered custom data collection enabled by smartphone apps an effective way to overcome the limitations of traditional $\mathrm{VoC}$ techniques. The design and market researchers in our study used MMRAs to overcome the constraints of traditional research methods and enable big data approaches that drew clients closer to customers. In all of these cases, the MMRA provided rich data, empowered customers, and helped the client move beyond firmcentric VoC processes, as called for by Goffin et al. (2012).

MMRA approaches are particularly effective in the early stages of the innovation process, when ideas are emerging and identification of hidden needs can be most effective in guiding development. An effective app gives customers the opportunity to voice their experiences in settings normally out of bounds to researchers. The responsiveness and real-time nature of MMRA data collection allows emerging issues to be probed and nascent concepts to be tested on the fly.

But MMRAs are not a panacea. To fully exploit the potential of MMRAs, market researchers and innovation managers must be aware of the effect the rigidities of legacy organizational structures can have on the MMRA process and the data analysis. Traditional divisions of tasks, between junior and senior researchers or between qualitative and quantitative researchers, may have to give way to the demands of the MMRA data-collection process. Indeed, some tasks, and some kinds of data, may require specialist processing and support; market researchers will have to collaborate with external partners, departing from the traditional supplier relationship. Such changes to traditional working practices do not come without their own issues - notably stress and uncertainty and the need for researchers to develop new skills. Managers should support team members in learning how to design appropriate MMRA tasks and interpret the large amounts of rich data MMRAs produce. 


\section{Conclusion}

MMRAs can facilitate innovation and design breakthroughs by allowing innovation teams to access rich, intimate data on customers and empowering customers to freely voice their experiences. Further, MMRAs can reach beyond the constraints of traditional research methods and enable innovative big data approaches that allow researchers and innovators to get closer to the authentic voice of the customer. However, MMRAs are not just a cheaper way to collect traditional data. They come with their own challenges and may demand new approaches and new skills, which innovation and design teams must address.

Research in this article was supported in part by the European Regional Development Fund.

\section{References}

Cluley, R. and Green, W. 2019. Social representations of marketing work: Advertising workers and social media. European Journal of Marketing 53(5): 830-847.

Cooper, R., and Edgett, S. 2008. Ideation for product innovation: What are the best methods? PDMA Visions Magazine 32(1): 12-17.

Davila, T., Epstein, M., and Shelton, R. 2012. Making Innovation Work: How to Manage It, Measure It, and Profit from It. Updated ed. Upper Saddle River, NJ: Pearson Education.

George, G., Haas, M. R., and Pentland, A. 2014. Big data and management. Academy of Management Journal 57(2): 321-326.

Gobble, M. M. 2015. From customer intelligence to customer intimacy. Resources. Research-Technology Management 58(6): 56-61.

Goffin, K., Lemke, F., and Koners, U. 2010. Identifying Hidden Needs: Creating Breakthrough Products. London: Palgrave Macmillan.

Goffin, K., Varnes, C., van der Hoven, C., and Koners, U. 2012. Beyond the voice of the customer. Research-Technology Management 55(4): 45-53.

Green, W. and Cluley, R. 2014. The field of radical innovation: Making sense of organizational cultures and radical innovation. Industrial Marketing Management 43(8): 1343-1350.

Kitchin, R. 2014. Big data, new epistemologies and paradigm shifts. Big Data \& Society 1(1): 1-12. 


\section{Text box}

\section{Case 1: Exploring Travelers' Experiences to Drive an Airline's Strategic Design Decisions}

In this case, presented by an innovation manager at the research organization, an MMRA was the main data collection method for capturing $\mathrm{VoC}$ data. The client was an airline that had implemented an ongoing $\mathrm{VoC}$ process to understand customers' travel experiences. Previous studies had focused on specific segments of a typical journeyarriving at the airport, boarding the plane, the in-flight experience-but the airline now wished to collect a more holistic view of the entire travel experience, from home through the airport to the destination (or vice versa). Traditional qualitative VoC methods would be challenging - if not impossible - in this context, as they would require following passengers for their entire journeys: the commute from home to the airport, via roads, parking lots, train stations, or taxis; the passage through airport security checks; the preflight time passed in cafés, duty-free shops, and lounges and waiting areas; and the boarding experience - as well as their use of online booking tools and their experiences of delays and interactions with ground personnel, airline cabin crew, and other passengers. And, the VoC research needed to collect the same information about the customer's experiences at the destination airport, from deplaning to baggage control to the commute to a hotel or final destination. That ethnographic study would need to be followed by a survey and possible researcher interviews with the travelers. The complexity of this effort was compounded by the need to study different routes, at different times, with different types of passengers and classes of travel.

An MMRA was used to overcome these challenges. Passengers on a number of flights were asked to download the custom-designed app. Throughout their travel experiences, participants were prompted to take pictures or videos, complete brief surveys, or log comments about the experience. The MMRA allowed researchers to gather data continually across private and public spaces and in a variety of forms, including videos, soundbites, and photographs of real customer experiences. These artifacts allowed market researchers to follow each passenger virtually and provided rich data that represented customers' full experiences. The research firm's client, the airline company, used the final report to redesign its passenger services.

In this case, data from the MMRA challenged the client's initial thinking. The client had sought to standardize its product offering across countries and passenger types, but the data from the large number of passenger journeys for which MMRA data were collected revealed real differences among passengers and between markets, not only in the way passengers behaved in airports but also in how they prepared for their journeys and made their way to and from the airport. This discovery led the client to shift its focus from standardizing to personalizing. The interviewee who described this case observed that while the MMRA data did "not fit into our neat way of thinking . . . it was great. It meant that we had a lot of data." 


\section{Text box}

\section{Case 2: Reconceptualizing the Experiences of Coffee Drinkers}

The aim of this project, which was described to the researchers by a digital research manager at the research organization, was to use VoC methods to reconceptualize coffee consumption as part of an effort to redesign the coffee cup. The client was a global coffee-shop chain seeking VoC data to feed into a design workshop that would include both designers and customers. In this case, MMRA was used to complement (rather than replace) traditional VoC methods. The interviewee explained that the project involved asking respondents to "give us their thoughts, give us examples, take photos, take videos, whatever it may be." The MMRA designed for the project allowed customers the freedom to represent their experiences as they wished. The MMRA offered a way to gain insights from customers without the researcher intervening in - and perhaps disrupting their experiences.

Not only did the data provide a new lens on customers' experience in the client's shops; they also allowed a wider team to engage with the data. The data collected by the app could be shared quickly and efficiently, and nearly in real time, through the app itself. As a result, the client's strategic design teams could research all of their global markets concurrently. That ability provided the MMRA's main advantage, from the interviewee's point of view. As the interviewee explained, it had previously been "basically impossible to have a single [research team]" analyzing such data. Rather, the client had had to rely on "secondary reportage or analysis . . . by other researchers." Thus, the MMRA provided the client an authentic customer voice across global markets, unmediated by third-party research or analysts. 


\section{Text box}

\section{Case 3: Mapping Children's Experience of Toothbrushing}

The aim of this project, described to researchers by a digital research manager at the research organization, was to support the design of a new toothbrush for children that would change the experience for children and encourage them to brush properly. VoC data had been difficult to gather in this context because it was difficult for researchers to access the very private spaces of bathrooms and personal hygiene rituals. Moreover, the younger toothbrush users the client sought to study typically only had a voice through their parents and guardians.

An MMRA allowed the client to overcome these constraints and enter customers' homes through participant-generated video footage. With appropriate consent from parents and guardians, the app allowed children to document their morning and evening teethcleaning routines. The results were rich, as the interviewee explained: "We got back a lot of good stuff of people recording themselves brushing their teeth in the morning, talking about what the process was, recording a video before and after a shower, what products they used, that kind of stuff." The app also engaged participants through gamified surveys and real-time video questions.

The scalability of the MMRA allowed researchers to access these experiences across different households and family structures. In this case, the study was successful because the MMRA methodology forced the client and research team to collaborate to find ways to unlock the data the client required. This project challenged all participants to think outside the constraints of traditional research protocols. The research team and the client collaborated to create specific tasks, facilitated by the MMRA, around their research questions, rather than relying on standard questionnaires and interviews. This process resulted in an analysis that communicated effectively the challenges parents face in motivating their children to clean their teeth; those findings were used by the client company to stimulate new product ideas. 
Figure captions

Figure 1.- Representative screen from an MMRA 